\title{
ON THE IRREGULARITY OF THE IMAGE OF THE IITAKA FIBRATION
}

\author{
JUNGKAI A. CHEN AND CHRISTOPHER D. HACON
}

\begin{abstract}
We characterize the irregularity of the image of the Iitaka fibration in terms of the dimension of certain cohomological support loci.
\end{abstract}

\section{INTRODUCTION}

Let $L$ be a Cartier divisor on a projective variety $X$ with $\kappa(X, L) \geq 0$. The Iitaka fibration associated to $L$ is a birational model of the map induced by the linear series $|m L|$ for $m$ sufficiently big and divisible. We will denote it by $f_{L}: X^{\prime} \rightarrow Y_{L}$, where $X^{\prime}$ is an appropriate birational model of $X$. It is a fundamental tool in the birational classification of higher dimensional varieties. Therefore, it is important to understand the geometry of the image of the Iitaka fibration.

In this note, we characterize the irregularity of $Y_{L}$ in terms of dimension of certain cohomological support loci. To be more precise, let

$$
V_{m}(L)=V_{m}:=\left\{P \in \operatorname{Pic}^{0}(X) \mid h^{0}\left(X, L^{\otimes m} \otimes P\right) \neq 0\right\},
$$

and $V=\cup_{m \geq 1} V_{m}$. We show that the maximal irreducible component of $V$ passing through the origin, denoted by $G$, is in fact the subgroup $f^{*} \operatorname{Pic}^{0}\left(Y_{L}\right) \subset \operatorname{Pic}^{0}(X)$. In particular, $q\left(Y_{L}\right)=\operatorname{dim} G$.

In general, the locus $V$ need not be a group, it could have infinitely many irreducible components and there may exists $P, Q \in \operatorname{Pic}^{0}(X)$ such that $f_{L \otimes P}$ and $f_{L \otimes Q}$ are not birational. Nevertheless, we show that when one considers the case of the canonical divisor $L=K_{X}$, then the set $V\left(K_{X}\right)$ is a subgroup of $\mathrm{Pic}^{0}(X)$ such that its components consist of finitely many torsion translates of $\operatorname{Pic}^{0}(Y)$. Moreover, we show that, for all $P \in V$, one has that the Iitaka fibration $f_{K_{X} \otimes P}$ is birational to $f_{K_{X}}$.

Finally, we study the case when $X$ is of maximal Albanese dimension. In [CH2], the authors have shown that the translates through the origin of the components of $V_{1}\left(K_{X}\right)$ generate $\operatorname{Pic}^{0}(X)$. In $\S 4$ we show that the locous $T$ given by the intersection of all (translates through the origin of the components of $V_{1}\left(K_{X}\right)$ ) plays a fundamental role in understanding the geometry of $X$. In particular we show that: Let $X$ be of general type and maximal Albanese dimension. If $P_{1}(X)=1$ then $q(X)=\operatorname{dim}(X)$. Moreover, we construct examples of such varieties. This answers a question of Kollár (cf. [Kol2], Conjecture (17.9.2)). 
Acknowledgement. The second author would like to thank R. Pardini for valuable conversations.

\section{Notation and conventions.}

(1.1) Throughout this paper, we work over the field of complex numbers $\mathbb{C}$. $X$ will always denote a projective variety.

(1.2) $|D|$ will denote the linear series associated to the divisor $D$. We do not distinguish between line bundles, linear equivalence classes of divisors and invertible sheaves. Let $|V| \subset|L|$ be a linear subsystem. A $\log$ resolution of $|V|$ is a proper birational morphism $\mu: X^{\prime} \longrightarrow X$ such that $X^{\prime}$ is smooth, $\mu^{*}|V|=|W|+F$, where $|W|$ is base point free and the union of support of $F$ and the exceptional set of $\mu$ is a divisor with normal crossings support.

(1.3) For a real number $a$, let $\lfloor a\rfloor$ be the largest integer $\leq a$ and $\lceil a\rceil$ be the smallest integer $\geq a$. For a $\mathbb{Q}$-divisor $D=\sum a_{i} D_{i}$, let $\lfloor D\rfloor=\sum\left\lfloor a_{i}\right\rfloor D_{i}$ and $\lceil D\rceil=\sum\left\lceil a_{i}\right\rceil D_{i}$.

(1.4) We will denote by $A(X)$ the Albanese variety of $X$, by $\operatorname{alb}_{X}$ : $X \longrightarrow A(X)$ the Albanese morphism. As usual $\operatorname{Pic}^{0}(X)$ is the abelian variety dual to $A(X)$ parameterizing all topologically trivial line bundles on $X . \operatorname{Pic}^{0}(X)_{\text {tors }}$ will denote the set of torsion elements in $\operatorname{Pic}^{0}(X)$. We will denote by $m: A \times A \longrightarrow A$ the group law of an abelian variety $A$. Given subsets $Z, W \subset A$, we define $Z+W:=$ image $\left(\left.m\right|_{Z \times W}: Z \times W \longrightarrow A\right)$.

$(1.5)$ Let $\mathcal{F}$ be a coherent sheaf on $X$, then $h^{i}(X, \mathcal{F})$ denotes the complex dimension of $H^{i}(X, \mathcal{F})$. In particular, the plurigenera $h^{0}\left(X, \omega_{X}^{\otimes m}\right)$ are denoted by $P_{m}(X)$ and the irregularity $h^{0}\left(X, \Omega_{X}^{1}\right)$ is denoted by $q(X)$.

(1.6) Let $L$ be a Cartier divisor on $X$. If $h^{0}(X, L)>0$, then there is a rational map $\phi_{|L|}: X \rightarrow \mathbb{P}\left(H^{0}(X, L)\right)$ defined by the sections of $L$. The Iitaka dimension of a line bundle $L$ is defined as

$$
\kappa(L):=\max \left\{\operatorname{dim} \phi_{|m L|}(X) ; m \in \mathbb{N}\right\} .
$$

If $|m L|=\emptyset$ for all $m>0$, we set $\kappa(L)=-\infty$. A nonsingular representative of the Iitaka fibration of $X$ is a morphism of smooth complex projective varieties $f_{L}^{\prime}=f^{\prime}: X^{\prime} \longrightarrow Y$ such that for all sufficiently big and divisible integers $m, f^{\prime}: X^{\prime} \longrightarrow Y$ is birational to $f_{|m L|}: X \rightarrow f_{|m L|}(X)$. It is characterized up to birational equivalence by the following properties:

i) $f^{\prime}: X^{\prime} \longrightarrow Y$ is an algebraic fiber space (i.e. it is surjective with connected fibers);

ii) $\operatorname{dim}(Y)=\kappa(L)$;

iii) $\kappa\left(X_{y}^{\prime}, L \mid X_{y}^{\prime}\right)=0$ (where $X_{y}^{\prime}$ is a generic geometric fiber of $f^{\prime}$ ).

There is a semi-group $N(L)=N(X, L):=\left\{m \in \mathbb{N} ; H^{0}\left(X, L^{\otimes m}\right) \neq\right.$ $0\}$. The exponent $e(L)$ is the greatest common divisor of all elements of $N(L)$. For all $m \gg 0$, one has that $m \in N(L)$ iff $e(L) \mid m$. 


\section{COHOMOLOGICAL SUPPORT LOCI}

Lemma 2.1. Let $X$ be a closed subvariety (reduced and irreducible) of an abelian variety $A$ passing through the origin. If $X$ is closed under the group law, then $X$ is an abelian subvariety.

Proof. Let $g$ be the dimension of $X$. Assume that $g>0$. Since $X$ is closed under multiplication, we have an induced morphism

$$
m: X \times X \rightarrow X \text {. }
$$

Let $F_{x}$ be the fiber over $x \in X$. It is clear that $\operatorname{dim} F_{x} \geq g$. On the other hand, one notices that $F_{x} \cap(\{a\} \times X)$ consists of at most one point for all $a \in X$. This, together with the fact that $\operatorname{dim} F_{x} \geq g$, shows that $F_{x} \cap(\{a\} \times X)$ has exactly one point for all $a \in X$. Let $x=0$ then it follows that $X$ is a subgroup of $A$ hence an abelian subvariety.

Let $X$ be a smooth projective variety with $q(X)>0, L$ a Cartier divisor on $X$ with $\kappa(L) \geq 0$ and exponent $e:=e(L)$. We define

$$
V_{m}=V_{m}(X, L):=\left\{P \in \operatorname{Pic}^{0}(X) \mid h^{0}\left(X, L^{\otimes m} \otimes P\right) \neq 0\right\} .
$$

By semi-continuity, each component of $V_{m}$ is a closed subset of $\operatorname{Pic}^{0}(X)$. Let $V=\cup_{m=1}^{\infty} V_{m}$. Then $V \subset \operatorname{Pic}^{0}(X)$ is a semi-group. We define $G_{m}$ to be the union of all irreducible components of $V_{m}$ passing through the origin and let $G=\cup_{m>0} G_{m}$. Note that $G_{m}$ is non-empty only when $m \in N(L)$. Recall that for all $m \gg 0$, we have $m \in N(L)$ iff $e(L) \mid m$.

Lemma 2.2. There exists an integer $m>0$ such that $G=G_{m}$ is an abelian subvariety of $\operatorname{Pic}^{0}(X)$.

Proof. Pick any maximal irreducible component $W \subset G$, that is, if $W^{\prime}$ is an irreducible component of $G$ containing $W$ then $W=W^{\prime}$. (This is possible since $\operatorname{Pic}^{0}(X)$ is of course Noetherian). Assume that $W \subset G_{m_{0}}$. We claim that $W=G$ and $W$ is closed under multiplication. Then we are done by Lemma 2.1. To see the claim, observe that if $Z$ is any irreducible component of $G_{m}$, then $Z+W$ is irreducible since it is the image of $m: Z \times W \rightarrow \operatorname{Pic}^{0}(X)$. Moreover,

$$
Z+W \subset G_{m_{0}}+G_{m} \subset G_{m_{0}+m} \subset G .
$$

Let $W^{\prime}$ be an irreducible component of $G_{m_{0}+m}$ containing $Z+W$. It follows that $W \subset Z+W \subset W^{\prime}$. By the maximality of $W$, one has $W=W^{\prime}$. In particular, $Z \subset W$ and hence $W=G$.

It then suffices to check that $W$ is closed under multiplication. But $W \subset W+W \subset G+G=G=W$ and hence $W+W=W$.

Corollary 2.3. There exists an integer $t_{0}>0$ such that $G_{t e}=G$ for all $t \geq t_{0}$.

Question: Are the loci $V$ (resp. $V_{m}$ ) union of translates of subgroups of $\operatorname{Pic}^{0}(X)$ ? 
Remark: It is easy to see that if $k(L)=0$ and $P \in V$ is a non torsion element, then $P^{\vee}$ is not in $V$. In particular, $\operatorname{dim} G=0$. The following example, shows that $\operatorname{dim} V_{m}$ could be positive even if $\kappa(L)=0$.

Example 2.4. Consider $A=E \times E$ a product of elliptic cures and let $M=p_{1}^{*} H+p_{2}^{*} P$ with $H$ ample of degree 1 and $P \in \operatorname{Pic}^{0}(E)-$ $\operatorname{Pic}^{0}(E)_{\text {tors }}$. Let $X=\mathbb{P}\left(\mathcal{O}_{A} \oplus M\right), \pi: X \longrightarrow A$ and $L:=\mathcal{O}_{X}(1)$. Since $\pi: X \longrightarrow A$ is a projective bundle, it follows that $\pi$ is the Albanese morphism of $X$. Since

$$
\pi_{*} \mathcal{O}_{X}(m)=S^{m}\left(M \oplus \mathcal{O}_{A}\right)=M^{\otimes m} \oplus M^{\otimes m-1} \oplus \ldots \oplus M \oplus \mathcal{O}_{A},
$$

one sees that

$$
V_{m}=\left\{\mathcal{O}_{A}\right\} \cup \bigcup_{i=1}^{m}\left(p_{1}^{*} \operatorname{Pic}^{0}(E)+i p_{2}^{*} P^{\vee}\right)
$$

We have

(1) $\kappa(L)=0$ and $\operatorname{dim} G(L)=0$.

(2) For all $m>0, Q \in\left(p_{1}^{*} \operatorname{Pic}^{0}(E)+m p_{2}^{*} P^{\vee}\right), \kappa(L \otimes Q)=1$ and $\operatorname{dim} V_{m}=\operatorname{dim} V=1$.

This gives an example in which $\kappa(L)=0$ but $\kappa(L \otimes P)>0$ for some $P \in \operatorname{Pic}^{0}(X)$. Moreover, $V=\cup V_{m}$ is a semi-group (with infinitely many components) but not a group.

Consider the following diagram relating the Iitaka fibration $f_{L}$ and the Albanese morphism alb ${ }_{X}$

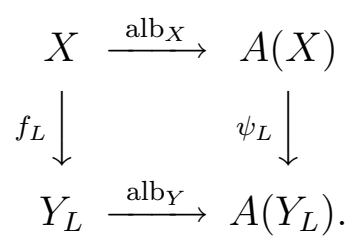

Notice that $f_{L}$ is not a morphism but simply a rational map. However there exists a birational model $\mu: X^{\prime} \longrightarrow X$ such that the induced map $f_{L}^{\prime}: X^{\prime} \longrightarrow Y_{L}$ is a morphism. Let $\pi_{L}: X^{\prime} \longrightarrow A\left(Y_{L}\right)$ be the induced map.

Lemma 2.5. If $P \in V_{m}$ then for all sufficiently big and divisible integers, $s>0$ one has that $s P+\operatorname{Pic}^{0}\left(Y_{L^{\otimes m} \otimes P}\right) \subset V$. In particular, if $\kappa(L) \geq 0$, then $\operatorname{Pic}^{0}\left(Y_{L}\right) \subset G(L)$

Proof. Let $N=L^{\otimes m} \otimes P$. Fix $H$ an ample line bundle on $A\left(Y_{L^{\otimes m} \otimes P}\right)$. For $k \gg 0$ sufficiently divisible, we have that $h^{0}\left(X^{\prime}, \mu^{*} N^{\otimes k} \otimes \pi^{*} H^{\vee}\right)>$ 0 . Clearly, $\mu_{*} \mathcal{O}_{X^{\prime}}=\mathcal{O}_{X}$ and $R^{i} \mu_{*} \mathcal{O}_{X^{\prime}}=0$ for all $i>0$. Since $\mu^{*} N^{\otimes k} \otimes \pi_{N}^{*} H^{\vee}=\mu^{*}\left(N^{\otimes k} \otimes \mathrm{alb}_{X}^{*} \psi_{N}^{*} H^{\vee}\right)$, by the projection formula, we have $h^{0}\left(X, N^{\otimes k} \otimes \operatorname{alb}_{X}^{*} \psi_{N}^{*} H^{\vee}\right)>0$. It is easy to see that for any integer $j \gg 0$, one has $h^{0}\left(H^{j} \otimes Q\right)>0$ for any $Q \in \operatorname{Pic}^{0}\left(Y_{L_{\otimes m} \otimes P}\right)$. It follows that $h^{0}\left(X, N^{\otimes k j} \otimes Q^{\otimes j}\right)>0$, i.e. $k j P+j Q \in V_{m k j}$. The Lemma follows by setting $s=k j$. 
In particular, take $P=\mathcal{O}_{X} \in V_{m}$, then $\mathcal{O}_{X} \in \operatorname{Pic}^{0}\left(Y_{L}\right) \subset V_{m k j}$ and hence $\operatorname{Pic}^{0}\left(Y_{L}\right) \subset G$.

It follows that for any $m>0$ and $P \in \operatorname{Pic}^{0}(X)$, we have $q\left(Y_{L \otimes P}\right) \leq$ $\operatorname{dim} G(L \otimes P) \leq \operatorname{dim} V(L)$. Recall though that it is possible $\operatorname{dim} V(L)>$ $G(L)$.

It also follows that if $L$ is big, then there exists an integer $m_{0}>0$ such that $V_{m}=\operatorname{Pic}^{0}(X)$ for all $m \geq m_{0}$.

Lemma 2.6. i) $\psi: A(X) \longrightarrow A(Y)$ is surjective and has connected fibers.

ii) Let $K:=k e r(\psi)$. Then there is an exact sequence

$$
0 \longrightarrow \operatorname{Pic}^{0}(Y) \longrightarrow \operatorname{Pic}^{0}(X) \longrightarrow \operatorname{Pic}^{0}(K) \longrightarrow 0 .
$$

iii) Fix a general fiber $i: X_{y} \longrightarrow X$. Let

$$
H:=\operatorname{ker}\left(i^{*}: \operatorname{Pic}^{0}(X) \longrightarrow \operatorname{Pic}^{0}\left(X_{y}\right)\right) \text {. }
$$

Then, $H \supset \operatorname{Pic}^{0}(Y)$ and $H / \operatorname{Pic}^{0}(Y)$ is a finite group.

Proof. i) See [HP] Proposition 2.1.b.

ii) This is an immediate consequence of i) obtained by dualizing the exact sequence of abelian varieties

$$
0 \longrightarrow K \longrightarrow A(X) \longrightarrow A(Y) \longrightarrow 0 .
$$

iii) Let $J_{y}$ be the abelian subvariety generated by the translates of $\operatorname{alb}_{X}\left(X_{y}\right)$ through origin. Since there are at most countably many sub-abelian varieties of a fixed abelian variety, we have that $J=J_{y}$ does not depend on $y \in Y$ for general $y$. Let $B:=A(X) / J$. Then $Y \longrightarrow A(Y)$ factors through $B$, and hence there is a map of abelian varieties $A(Y) \longrightarrow B$ inducing the map $Y \longrightarrow B$. The image of $X$ in $A(X)$ generates $A(X)$, hence its image in $B$ generates $B$ as well. It follows that image of $A(Y)$ in $B$ generates $B$. And hence, one sees that $B=A(Y)$ i.e. $K=J$.

We have the following commutative diagram

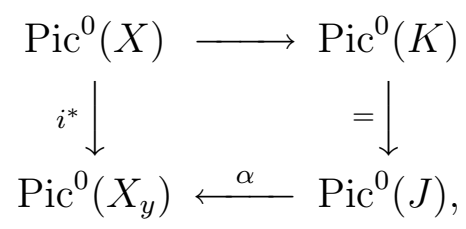

where $i: X_{y} \rightarrow X$ is the inclusion and $\alpha$ is induced from alb $\operatorname{al}_{X}: X_{y} \rightarrow J$. Note that, since $A\left(X_{y}\right) \rightarrow J$ is surjective, the homomorphism $\alpha$ has finite kernel. The assertion now follows from ii).

Theorem 2.7. $G(L)=\operatorname{Pic}^{0}\left(Y_{L}\right)$. 
Proof. By Lemma 2.5, one has $G(L) \supset \operatorname{Pic}^{0}\left(Y_{L}\right)$.

If $\kappa(L)=0$, it suffices to prove that $\operatorname{dim} G=0$. This is clear as otherwise one may consider $m>0$ such that $G(L) \subset V_{m}$ and the morphisms

$$
|m L+P| \times|m L-P| \longrightarrow|2 m L|
$$

for $P \in G$. It is easy to see that if $\operatorname{dim} G>0$ then $\operatorname{dim}|2 m L|>0$ a contradiction.

Assume now that $\kappa(L)>0$. If $P \in G(L)$, then for some $m \gg 0$, $h^{0}\left(L^{\otimes m} \otimes P\right) \neq 0$ in particular $h^{0}\left(X_{y}, L^{\otimes m} \otimes P \mid X_{y}\right) \neq 0$. It follows that $i^{*} G(X, L) \subset V_{m}\left(X_{y}, L \mid X_{y}\right)$. And hence $i^{*} G(X, L) \subset G\left(X_{y}, L \mid X_{y}\right)$. However, $G\left(X_{y}, L \mid X_{y}\right)=\left\{\mathcal{O}_{X_{y}}\right\}$ as we have seen in the preceding paragraph. Thus we have

$$
i^{*} G(X, L)=G\left(X_{y}, L \mid X_{y}\right)=\left\{\mathcal{O}_{X_{y}}\right\} .
$$

By Lemma 2.6, $G(X, L) \subset H$. Since $G(X, L)$ is connected and contains the origin, it follows that $G(X, L) \subset \operatorname{Pic}^{0}\left(Y_{L}\right)$.

\section{IITAKA FIBRATIONS OF CANONICAL DIVISORS}

¿From now on we will assume $L=K_{X}$ and $\kappa(X) \geq 0$.

Proposition 3.1. Fix an integer $m \geq 2$. For all $Q \in \operatorname{Pic}^{0}(X)_{\text {tors }}$ and all $P \in \operatorname{Pic}^{0}\left(Y_{K_{X}}\right)$ one has

$$
h^{0}\left(\omega_{X}^{\otimes m} \otimes P \otimes Q\right)=h^{0}\left(\omega_{X}^{\otimes m} \otimes Q\right) .
$$

Proof. Just follow the proof of [HP] Proposition 2.12.

Theorem 3.2. The loci $V_{m}\left(K_{X}\right)$ consist of a finite union of torsion translates of abelian subvarieties of $\operatorname{Pic}^{0}(X)$.

Proof. By a result of Simpson, the loci $V_{1}\left(K_{X}\right)$ are torsion translates of abelian subvarieties of $\operatorname{Pic}^{0}(X)$. Fix $m \geq 2$, and let $P \in V_{m}\left(K_{X}\right)$. Let $\mu: X^{\prime} \longrightarrow X$ be a log resolution of the non empty linear series $\left|r\left((m-1) K_{X}+P\right)\right|$. Let $D$ be a general member of $\mu^{*}\left|r\left((m-1) K_{X}+P\right)\right|$. $D$ has normal crossings support. Let $N:=\mathcal{O}_{X^{\prime}}\left((m-1) K_{X^{\prime}}+P-\left\lfloor\frac{D}{r}\right\rfloor\right)$, then the locus

$$
V_{1}\left(\omega_{X^{\prime}} \otimes N\right):=\left\{Q \in \operatorname{Pic}^{0}(X) \mid h^{0}\left(\omega_{X^{\prime}} \otimes N \otimes Q\right)>0\right\}
$$

consist of a finite union of torsion translates of subtori of $\operatorname{Pic}^{0}(X)$ (cf. [Sim] and $[\mathrm{ClH}] \S 7$ ). Comparing base loci, it is easy to see that $h^{0}\left(\omega_{X^{\prime}} \otimes N\right)=h^{0}\left(\omega_{X^{\prime}}^{\otimes m} \otimes P\right)$ and for all $Q \in T_{P}$ we have

$$
h^{0}\left(\omega_{X^{\prime}}^{\otimes m} \otimes Q\right)=h^{0}\left(\omega_{X^{\prime}}\left(\left\lfloor\frac{D}{r}\right\rfloor\right) \otimes N \otimes Q \otimes P^{\vee}\right) \geq h^{0}\left(\omega_{X^{\prime}} \otimes N \otimes Q \otimes P^{\vee}\right)>0 .
$$

Hence for each $P \in V_{m}\left(K_{X}\right)$ there exists $T_{P}$ a torsion translate of a subtorous such that $P \in T_{P} \subset V_{m}\left(K_{X}\right) \subset \operatorname{Pic}^{0}(X)$. It follows that $V_{m}\left(K_{X}\right)=\cup T_{P}$. Since $V_{m}\left(K_{X}\right) \subset \operatorname{Pic}^{0}(X)$ is closed, it follows that $V_{m}\left(K_{X}\right)$ consists of a finite union of the $T_{P}$. 
Lemma 3.3. If $\kappa(X)=0$ then there exists an integer $c(X)=c>0$ and an element $P \in \operatorname{Pic}^{0}(X)_{\text {tors }}$ such that for all $m>0$, the set $V_{m}\left(K_{X}\right)$ is non-empty if and only if $c$ divides $m$. Moreover, if $c$ divides $m$, then $V_{m}=\left\{P^{\otimes \frac{m}{c}}\right\}$. In particular $V$ is a subgroup of $\operatorname{Pic}^{0}(X)$.

Proof. It is easy to see that since $\kappa(X)=0$, each $V_{m}$ can have at most 1 torsion point. Therefore $V_{m}\left(K_{X}\right)$ consists of at most one element say $P_{m} \in \operatorname{Pic}^{0}(X)_{\text {tors }}$. Let

$$
c:=\min \left\{m>0 \mid V_{m} \neq \emptyset\right\} .
$$

It is clear that $V_{m} \neq \emptyset$ for all $m$ divisible by $c$. Let $d$ be any positive integer such that $V_{d} \neq \emptyset$. We claim that $c \mid d$. We may write $d=c q+r$ with $q>0$ and $0 \leq r \leq c-1$. Let $D_{c}$ (resp. $D_{d}$ ) be the unique divisor in the linear series $\left|c K_{X}+P_{c}\right|$ (resp. $\left|d K_{X}+P_{d}\right|$ ). Since $V_{c d}$ has only one point, we have $d P_{c}=c P_{d}$ and moreover $c D_{d}=d D_{c}$ as $h^{0}\left(c d K_{X}+d P_{c}\right)=1$.

Consider now the divisor $D_{d}-q D_{c}$. It is effective since $c\left(D_{d}-q D_{c}\right)=$ $r D_{c}$ is effective. It follows that $h^{0}\left(r K_{X}+P_{d}-q P_{c}\right) \neq 0$ and hence $V_{r} \neq \emptyset$. One sees that $r=0$. Therefore, $V_{m}\left(K_{X}\right)$ is non-empty iff $c$ divides $m$. The Lemma now follows easily.

Corollary 3.4. Let $\widetilde{H}:=i^{*-1}\left(V\left(X_{y}\right)\right)$. For all $m>0$ one has $V_{m} \subset$ $\widetilde{H}$. Moreover, $V \subset \widetilde{H}$ is a subgroup of finite index containing $G$.

Proof. For any $Q \in V_{m}$, if $h^{0}\left(\omega_{X}^{\otimes m} \otimes Q\right) \neq 0$, then $h^{0}\left(\omega_{X_{y}}^{\otimes m} \otimes Q\right) \neq 0$. Therefore, $V_{m} \subset \widetilde{H}$ for all $m>0$ and hence, $V \subset \widetilde{H}$.

Since $\widetilde{H} / G$ is finite and $G \subset V \subset \widetilde{H}$, it suffices to show that the semigroup $V$ is in fact a group.

To this end, pick any $P \in V$, we may assume that $P \in V_{m}$ for $m \geq 2$ since $V_{1} \subset V_{1+t}$ for all sufficiently big and divisible $t$. By Proposition 3.1 and Theorem 3.2, one sees that $P$ is in an irreducible component of the type $G+P_{0}$ with $P_{0}$ torsion. Let $k$ be the order of $P_{0}$. One sees that

$$
-P \subset G-P_{0}=(k-1)\left(G+P_{0}\right) \subset V .
$$

It follows that $V$ is a group.

\section{Corollary 3.5.}

$$
q(X)-q(Y) \leq q\left(X_{y}\right)
$$

Proof. Since $\kappa\left(X_{y}\right)=0$, by [Kaw], the map alb $X_{y}: X_{y} \longrightarrow A\left(X_{y}\right)$ is surjective. The map $X_{y} \longrightarrow J$ is factored through $A\left(X_{y}\right)$. It follows that $A\left(X_{y}\right) \longrightarrow J$ is surjective by the definition of $J$. Thus $q\left(X_{y}\right)=$ $\operatorname{dim} A\left(X_{y}\right) \geq \operatorname{dim} J$. But $J=K$ and $\operatorname{dim} K=\operatorname{dim} A(X)-\operatorname{dim} A(Y)$. The inequality follows.

Corollary 3.6. $X$ is of maximal Albanese dimension if and only if $Y$ is of maximal Albanese dimension and

$$
q(X)-q(Y)=\operatorname{dim} X-\operatorname{dim} Y .
$$


Proof. If $X$ is of maximal Albanese dimension, then alb $_{X}: X \rightarrow A(X)$ is generically finite. It follows that $\mathrm{alb}_{X}: X_{y} \rightarrow A(X)$ is generically finite. Therefore, $X_{y} \longrightarrow J$ is an étale map of abelian varieties and $\operatorname{dim} X_{y}=\operatorname{dim} J$. Moreover, since alb ${ }_{X}$ is generically finite, for a general point $z \in \operatorname{alb}_{Y}(Y)$, the preimage in $X$ consits of at most finite union of fibers $X_{y}$. Thus alb Th $_{Y}$ is generically finite and hence $Y$ is of maximal Albanese dimension.

On the other hand, let $Y \rightarrow S \rightarrow A(Y)$ be the Stein factorization. And let $T:=S \times_{A(Y)} A(X)$. It suffices to check that $X \rightarrow T$ is generically finite. Notice that $\kappa\left(X_{y}\right)=0$ and $q\left(X_{y}\right) \geq \operatorname{dim} X_{y}$. It follows by [Kaw] that $X_{y}$ is birational to an abelian variety and hence that $X_{y} \rightarrow K$ is generically finite. Therefore $X_{y} \rightarrow T$ is generically finite, and so is $X \rightarrow T$.

Corollary 3.7. For all $P \in \operatorname{Pic}^{0}(Y)$, the Iitaka fibration $Y\left(K_{X}+P\right)$ is birational to the Iitaka fibration $Y\left(K_{X}\right)$.

Proof. Let $P, Q \in \operatorname{Pic}^{0}(Y)$ such that $P=2 Q$. From the morphism

$$
\left|m K_{X}\right| \times\left|m\left(K_{X}+P\right)\right| \longrightarrow\left|2 m\left(K_{X}+Q\right)\right|,
$$

one sees that $Y\left(K_{X}+Q\right)$ dominates $Y\left(K_{X}\right)$ and $Y\left(K_{X}+P\right)$. By Proposition 3.1,

$$
\operatorname{dim} Y\left(K_{X}+Q\right)=\operatorname{dim} Y\left(K_{X}+P\right)=\operatorname{dim} Y\left(K_{X}\right) .
$$

since the various Iitaka fibrations $X \longrightarrow Y(\ldots)$ have connected fibers, then $Y\left(K_{X}+Q\right)$ is birational to $Y\left(K_{X}\right)$ and to $Y\left(K_{X}+P\right)$.

Lemma 3.8. Let $a: X \rightarrow A(X)$ be the Albanese map. If $q(Y)=0$, then $a_{*} \omega_{X}$ is a homogeneous vector bundle. In particular, $c_{1}\left(a_{*} \omega_{X}\right)=$ 0 .

Proof. Let $a=\operatorname{alb}_{X}$. If $q(Y)=0$, then $V_{1}$ is supported on finitely many points. We recall that by definition

$$
V^{i}\left(a_{*} \omega_{X}\right):=\left\{P \in \operatorname{Pic}^{0}(A(X)) \mid h^{i}\left(a_{*} \omega_{X} \otimes P\right) \neq 0\right\} .
$$

By [CH1], we have

$$
V_{1}=V^{0} \supseteq V^{1} \supseteq \ldots \supseteq V^{q},
$$

where $q=q(X)$. It follows that all the $V^{i}$ are supported on finitely many points.

We now follow [Laz] to show that $a_{*} \omega_{X}$ is a homogeneous vector bundle. Let $p_{i}$ be the projection of $A(X) \times \operatorname{Pic}(X)=A \times \widehat{A}$ on to the $i$-th factor and $\mathcal{P}$ be the normalized Poincare' line bundle on $A \times \widehat{A}$. Then $R S a_{*} \omega_{X}=R p_{2 *}\left(p_{1}^{*} a_{*} \omega_{X} \otimes \mathcal{P}\right)$ is the Fourier-Mukai transform of $a_{*} \omega_{X}$. By [Muk], it is enough to show that $R^{g} S a_{*} \omega_{X} \cong R S a_{*} \omega_{X}$ is a coherent sheaf supported on finitely many points. To see this, recall 
that for an open affine $U=\operatorname{Spec}(B) \subset \hat{A}$, there is a complex of finitely generated free $B$-modules, denoted by $E^{\bullet}$, such that

$$
R^{i} S a_{*} \omega_{X} \cong H^{i}\left(E^{\bullet}\right),
$$

and

$$
H^{i}\left(X_{y}, a_{*} \omega_{X} \otimes P_{y}\right) \cong H^{i}\left(E^{\bullet} \otimes k(y)\right) .
$$

It suffices to show that $H^{i}\left(E^{\bullet}\right)=0$ for all $i<q$ and $H^{q}\left(E^{\bullet}\right)$ is supported on finite point. We may assume that $B$ is local since localization is flat. Let

$$
W^{i}\left(E^{\bullet}\right):=\operatorname{coker}\left(E^{i-1} \rightarrow E^{i}\right) .
$$

It easy to see that $W^{i}\left(E^{\bullet}\right) \otimes k(y)=W^{i}\left(E^{\bullet} \otimes k(y)\right)$ since $\otimes$ is right exact. One has exact sequence

$$
0 \rightarrow H^{i}\left(E^{\bullet}\right) \rightarrow W^{i}\left(E^{\bullet}\right) \rightarrow E^{i+1}
$$

Our hypothesis on the cohomologies of $a_{*} \omega_{X}$ implies that $H^{i}\left(E^{\bullet}\right)$ is Artinian for all $i$. Thus by Mumford's acyclic lemma ([Mum], page 127), we are done.

\section{Varieties of Maximal Albanese dimension}

Throughout this section, we assume that $X$ is of maximal Albanese dimension i.e. $\operatorname{dim}\left(\operatorname{alb}_{X}(X)\right)=\operatorname{dim} X$.

In (17.9.3) of [Kol2], Kollár Conjectured that If $X$ is of general type and Maximal Albanese dimension, then $\chi\left(X, \mathcal{O}_{X}\right)>0$. A consequence of this conjecture would be:

i) $V_{1}\left(X, K_{X}\right)=\operatorname{Pic}^{0}(X)$ and

ii) (cf. (17.9.2) of $[\mathrm{Kol} 2])$ that $P_{1}(X) \geq 2$.

In [EL], Ein and Lazarsfeld show that $\chi\left(X, \mathcal{O}_{X}\right) \geq 0$, and they produce a counterexample to the above conjecture of Kollár ((17.9.3) of [Kol2]). In [CH2], the authors show that:

Theorem 4.1. If $X$ is of maximal Albanese dimension then (the translates through the origin of) the irreducible components of $V_{1}\left(X, K_{X}\right)$ generate $\operatorname{Pic}^{0}(Y)$, and for all $m \geq 2$ one has $G_{m}\left(X, K_{X}\right)=\operatorname{Pic}^{0}(Y)$.

In particular if $X$ is of general type, they generate $\operatorname{Pic}^{0}(X)$ (see i) above).

Let $X$ be of maximal Albanese dimension. If $\chi\left(\mathcal{O}_{X}\right)=0$, let $S_{i}^{\prime}$ be the translates of the components of $V_{1}\left(X, K_{X}\right)$ through the origin and $T:=\cap S_{i}^{\prime}$.

Theorem 4.2. Let $X$ be a variety of general type, maximal Albanese dimension with $\chi\left(\omega_{X}\right)=0$. Let $A(X) \longrightarrow T^{\vee}$ be the corresponding map of abelian varieties, and $\pi: X \longrightarrow T^{\vee}$ the induced morphism. For general $w$ in $W:=\pi(X)$, one has that $\chi\left(\omega_{X_{w}}\right)=0$ and $\operatorname{alb}_{X}\left(X_{w}\right)$ is a translate of the abelian subvariety $\operatorname{ker}\left(A(X) \longrightarrow T^{\vee}\right)$. In particular, $\operatorname{dim} T \neq 1$ and if $\operatorname{dim} T=0$ then $q(X)=\operatorname{dim}(X)$. 
Proof. Recall that by [EL] Theorem 3, the Albanese image $Z:=\operatorname{alb}_{X}(X)$ $\subset \operatorname{Alb}(X)$ is fibered by tori. In fact [EL] shows the following: Let $S$ be any positive dimensional component of $V^{0}\left(\omega_{X}\right)=V_{1}\left(X, K_{X}\right)$. Consider the quotient map $\pi: A:=\operatorname{Alb}(X) \longrightarrow C$ dual to the inclusion $S \hookrightarrow \operatorname{Pic}^{0}(A)$. Let $g: X \longrightarrow C$ be the induced morphism, then $\operatorname{dim} g(X)=\operatorname{dim}(X)-(\operatorname{dim}(A)-\operatorname{dim}(C))$, i.e. $Z \longrightarrow g(X)$ is fibered by tori. By [CH2] Theorem 2.3, the translates through the origin of the components of $V_{1}\left(\omega_{X}\right)$ generate $\operatorname{Pic}^{0}(X) . X$ is of general type and hence not ruled by tori. Assume that the intersection of the translates through the origin of the components of $V_{1}\left(\omega_{X}\right)$ is just $\left\{\mathcal{O}_{X}\right\}$. Let $S_{i}$ be any component of $V_{1}\left(\omega_{X}\right)$ and $B_{i}$ be the kernel of the corresponding projection of abelian varieties $\pi_{i}: A \longrightarrow C_{i}:=S_{i}^{\vee}$. Then the union of the $B_{i}$ generates $A$. As observed above, by the result of [EL], $Z+B_{i}=Z$ and hence $Z=A$, i.e. $q(X)=\operatorname{dim} X$.

Suppose now that $\operatorname{dim} T>0$ and consider the following dual exact sequences of abelian varieties

$$
\begin{gathered}
0 \longrightarrow T \longrightarrow \operatorname{Pic}^{0}(X) \stackrel{\phi}{\longrightarrow} \operatorname{Pic}^{0}(X) / T \longrightarrow 0, \\
0 \longrightarrow F \longrightarrow A(X) \longrightarrow T^{\vee} \longrightarrow 0 .
\end{gathered}
$$

Let $\bar{S}_{i}:=S_{i} / T \subset \operatorname{Pic}^{0}(F)=\operatorname{Pic}^{0}(X) / T$ and $\pi: X \longrightarrow T^{\vee}$ the induced morphism. For any $P \in \operatorname{Pic}^{0}(X)_{\text {tors }}$ such that $P \notin V_{1}\left(X, K_{X}\right)$, one has that

$$
\phi^{-1}(\phi(P))=P+T \cap V_{1}\left(X, K_{X}\right)=\emptyset .
$$

So for all $Q \in T$ one has $h^{0}\left(X, \omega_{X} \otimes P \otimes \pi^{*} Q\right)=0$ and therefore also $h^{i}\left(X, \omega_{X} \otimes P \otimes \pi^{*} Q\right)=0$ for all $i \geq 0$. By a result of Kollár (cf. [Kol1], Corollary 3.3) one sees that for all $Q \in T$, the group $H^{i}\left(\pi_{*}\left(\omega_{X} \otimes P\right) \otimes Q\right)$ is a direct summand of the group $H^{i}\left(X, \omega_{X} \otimes P \otimes \pi^{*} Q\right)$ and hence also vanishes. By a result of Mukai (cf. [Muk]) it follows that $\pi_{*}\left(\omega_{X} \otimes P\right)=$ 0 . So for general $w \in W=\pi(X)$, one has $h^{0}\left(X_{w}, \omega_{X_{w}} \otimes P\right)=0$. Therefore, $\chi\left(\omega_{X_{w}}\right)=0$ and the locous

$$
V_{1}\left(X_{w}, K_{X_{w}}, \operatorname{Pic}^{0}(F)\right):=\left\{R \in \operatorname{Pic}^{0}(F) \mid h^{0}\left(\omega_{X_{w}} \otimes R\right)>0\right\}
$$

(which is determined by its torsion points) is a subset of $V_{1}\left(X, K_{X}\right) / T$. It follows that the intersection of the translates through the origin of the components of $V_{1}\left(X_{w}, K_{X_{w}}, \mathrm{Pic}^{0}(F)\right)$ is just $\left\{\mathcal{O}_{F}\right\}$. Therefore, arguing as in the preceeding paragraph, one sees that $\operatorname{alb}_{X}\left(X_{w}\right)=F$ is an abelian subvariety of $A(X)$.

Corollary 4.3. Let $X$ be a 3-fold of general type and maximal Albanese dimension. If $\chi\left(\omega_{X}\right)=0$ then $q(X)=\operatorname{dim} X$.

Proof. We may assume that $\operatorname{dim} X>\operatorname{dim} T>0$ and hence $X_{w}$ is of general type and has dimension 1 or 2 . By the classification theory of curves and surfaces $\chi\left(\omega_{X_{w}}\right)>0$. This is the required contradiction.

Corollary 4.4. Let $X$ be a variety of general type, maximal Albanese dimension. If $P_{1}(X)=1$ then $q(X)=\operatorname{dim} X$. 
Proof. If $P_{1}(X)=1$ then $\chi\left(\omega_{X}\right)=0$. Let $\tilde{W} \longrightarrow W$ be an appropriate desingularization (of $W=\pi(X)$ as above). Since $X$ is of maximal Albanese dimension, one has $P_{1}(\tilde{W})=1$ and hence since $W$ is a subvariety of the abelian variety $T^{\vee}$, it follows that $W=T^{\vee}$. Since $\operatorname{alb}_{X}\left(X_{w}\right)=F$ one sees that $\operatorname{alb}_{X}(X)=A(X)$ and hence $q(X)=\operatorname{dim}(X)$.

Corollary 4.5. Let $X$ be a variety of general type, maximal Albanese dimension with $\chi\left(\omega_{X}\right)=0$. If $\operatorname{dim} T=0$, then $h^{1}(X, P)=0$ for all $P \in \operatorname{Pic}^{0}(X)-\left\{\mathcal{O}_{X}\right\}$.

Proof. Assume that $h^{1}(X, P)>0$ for some $P \in \operatorname{Pic}^{0}(X)-\left\{\mathcal{O}_{X}\right\}$. By [Sim], we may assume that $P$ is torsion. Consider the étale cover $Y \longrightarrow X$ corresponding to the subgroup $<P>\subset \operatorname{Pic}^{0}(X)$. Clearly $Y$ is of general type, maximal Albanese dimension and $\chi\left(\omega_{Y}\right)=0$ so by the Theorem 4.2

$$
\operatorname{dim}(Y)=q(Y)=h^{1}\left(\mathcal{O}_{Y}\right)=\sum h^{1}\left(X, P^{\otimes i}\right) .
$$

In particular $h^{1}\left(X, P^{\otimes i}\right)=0$ for $i>0$.

The following example shows that Kollár's conjecture (17.9.2) of [Kol2] also fails:

Example. Let $d: D \longrightarrow E$ be the $\mathbb{Z}_{2}^{2}$ cover of an elliptic curve defined by $d_{*} \mathcal{O}_{D}=\mathcal{O}_{E} \oplus L^{\vee} \oplus P \oplus L^{\vee} \otimes P$ where $\operatorname{deg}(L)=1$ and $P$ is a non-zero 2-torsion element of $\operatorname{Pic}^{0}(E)$. Then $g(D)=3$ and we will denote by $l, p, l p$ the elements of $\mathbb{Z}_{2}^{2}$ whose eigensheves with eigenvalue 1 are $\mathcal{O}_{E}$ and $L^{\vee}, P, L^{\vee} \otimes P$ respectively. It follows that

$$
\delta: D \times D \times D \longrightarrow E \times E \times E
$$

is a $\mathbb{Z}_{2}^{6}$ cover. Let $X:=D \times D \times D / G$ where $G \cong \mathbb{Z}_{2}^{4}$ is the subgroup of $\mathbb{Z}_{2}^{6}$ generated by $\{(i d, p, l),(p, l, i d),(l, i d, p),(p, p, p)\}$. By a direct check, one can see that

$$
\left(\delta_{*} \mathcal{O}_{D \times D \times D}\right)^{G \cong}
$$

$\mathcal{O}_{E} \otimes \mathcal{O}_{E} \otimes \mathcal{O}_{E} \oplus L^{\vee} \otimes L^{\vee} \otimes P \otimes P \oplus P \otimes L^{\vee} \otimes L^{\vee} \otimes P \oplus L^{\vee} \otimes P \otimes P \otimes L^{\vee}$

The singularities of $X$ are of type $\frac{1}{2}(1,1,1)$ i.e. locally isomorphic to $\mathbb{C}^{3} /<(-1,-1,-1)>$. In particular all singularities are rational. Let $\tilde{X} \longrightarrow X$ be a resolution of the singularities of $X$ and $\nu: \tilde{X} \longrightarrow$ $E \times E \times E$ be the induced morphism. One can compute that

$\nu_{*}\left(\omega_{\tilde{X}}\right)=\mathcal{O}_{E} \otimes \mathcal{O}_{E} \otimes \mathcal{O}_{E} \oplus L \otimes L \otimes P \otimes P \oplus P \otimes L \otimes L \otimes P \oplus L \otimes P \otimes P \otimes L$.

Therefore, $\tilde{X}$ is a threefold of maximal Albanese dimension with $P_{1}(X)=1$ and $q(X)=3$.

We remark that this example easily generalizes to $\operatorname{dim} X \geq 3$. The product $X \times C$ with $C$ a curve of genus 2 , is a variety of general type and maximal Albanese dimension with $\chi\left(\omega_{X \times C}\right)=0$ and $5=q(X)>$ $\operatorname{dim} X=4$. Moreover, for any étale cover $\tilde{X} \longrightarrow X$ one also has 
$\chi(\tilde{X})=0$. One can then construct (infinitely many) examples where $P_{1}(\tilde{X})=P_{1}(X), q(\tilde{X})=q(X)$.

\section{REFERENCES}

[CH1] J. A. Chen, C. D. Hacon, On algebraic fiber spaces over varieties of maximal Albanese dimension, to appear in Duke Math. Jour.

[CH2] A. J. Chen, C. D. Hacon, Pluricanonical maps of varieties of maximal Albanese dimension, Math. Annalen 320 (2001) 2, 367-380.

$[\mathrm{ClH}] \mathrm{H}$. Clemens and C. D. Hacon, Deformations of the trivial line bundle and vanishing theorems, Preprint math.AG/0011244. To appear in Amer. Jour. Math.

[EL] L. Ein, R. Lazarsfeld, Singularities of theta divisors, and birational geometry of irregular varieties, Jour. AMS 10, 1 (1997), 243-258.

[HP] C. D. Hacon and R. Pardini, On the birational geometry of varieties of maximal Albanese dimension To appear in Jour. für die Reine Angew.

[Kaw] Y. Kawamata, Characterization of abelian varieties, Comp. Math. 41, (1981).

[Kol1] J. Kollàr, Higher direct images of dualizing sheaves II, Ann. Math. (1987) 124

[Kol2] J. Kollàr, Shafarevich Maps and Automorphic Forms, Princeton University Press (1995).

[Laz] R. Lazarsfeld, Personal communication

[Muk] S. Mukai, Duality between $D(X)$ and $D(\hat{X})$ with its application to Picard sheaves, Nagoya Math. Jour. (1981).

[Mum] D. Mumford, Abelian Varieties, Oxford University Press.

[Sim] Simpson, C., Subspaces of moduli spaces of rank one local systems., Ann. scient. École Norm. Sup. 26 (1993), 361-401. 